ISSN: 1560-9111

\title{
Comparación de la calidad de sellado de tres técnicas de obturación radicular a través del microscopio estereoscópico
}

\author{
Comparison of sealing quality of three root canal filling \\ techniques through stereoscopic microscope
}

\section{Resumen}

Objetivo: Este estudio es una evaluación in vitro del sellado tridimensional del sistema de conductos radiculares realizados con tres técnicas de obturación: Condensación Lateral, Técnica Hibrida de Tagger y Termoplástica con Beefill 2 en 1, por tercios radiculares en dientes inferiores. Metodología: Se utilizaron 30 primeras premolares inferiores las cuales fueron divididas aleatoriamente en tres grupos de 10 para cada técnica de obturación. Se realizó la limpieza, desinfección y conformación del conducto radicular. Para la técnica Condensación Lateral y Beefill 2 en 1 se prepararon con el Sistema Mtwo, y para la técnica híbrida de Tagger se preparó con la técnica Crown Down de la Universidad de Oregon. Se obturaron las muestras y se realizaron cortes transversales en tercio cervical $(3 \mathrm{~mm})$, medio $(6 \mathrm{~mm})$ y apical $(9 \mathrm{~mm})$, y fueron llevados al microscopio estereoscópico. Resultados: Se realizó el análisis estadístico del Chi cuadrado y la prueba de comparación de proporciones con el test $Z$ nivel de significancia del 5\%.Grupo 3 (condensación lateral) grupo 2 (Hibrida de Tagger) grupo 1 (Beefill 2 en 1). A nivel del tercio cervical no hubo diferencias estadísticamente significativa entre los tres sistemas, $\mathrm{X}^{2}=3.36$, $\mathrm{p}=0.186$, se evidenció la presencia de vacíos solo en los grupos 2 y 3 . A nivel del tercio medio $\mathrm{X}^{2}=6.67$ encontrándose diferencias estadísticamente significativa en la calidad del sellado de Beefill con respecto a los otros $\mathrm{p}=0.036$. A nivel del tercio apical $\mathrm{X}^{2}=8.9$ encontrándose diferencias estadísticamente significativa en la calidad del sellado de Beefill con respecto a los otros $\mathrm{p}=0.014$. Posteriormente se realizó la prueba de comparación de proporciones con el test $\mathrm{Z}$ confirmándose la significancia de los hallazgos La técnica termoplástica con Beefill 2 en 1, demostró una calidad de sellado óptimo, en los tres tercios observándose diferencias estadísticamente significativas en el tercio medio y apical $\mathrm{p}<0.05$.

Conclusión: La técnica termoplástica de ola continua con Beefill 2 en 1 presenta mejor calidad de sellado en los tres tercios, pues al ablandar la gutapercha por calor y al ser compactada, esta discurre por todo el conducto radicular.

Palabras clave: Calidad de sellado; Técnica de obturación radicular; Beefill; Tagger; Condensación lateral.

\begin{abstract}
Objective: This study is an in vitro evaluation of three-dimensional root canal sealing ability of three obturation techniques: Lateral condensation, Tagger's hybrid technique and thermoplastic Beefill 2 in 1 in mandible teeth. Methodology: it was used 30 first lower premolars which were randomly divided into three groups of 10 each. Root canals were cleaned, disinfected and shaping. The groups of Lateral condensation technique and Beefill 2 in 1 were prepared with Mtwo System, and Tagger's hybrid technique with the University of Oregon Crown Down technique. Samples were prepared and cross sections were performed in the third cervical $(3 \mathrm{~mm})$, medium $(6 \mathrm{~mm})$ and apical $(9 \mathrm{~mm})$, and then taken to the stereoscopic microscope. Results: Statistical analysis was performed with Chi square and comparison of proportions with the $\mathrm{Z}$ tests on $5 \%$ level of significance. Group 3 (lateral condensation), Group 2 (hybrid of Tagger), Group 1 (Beefill 2 in 1). In cervical third there were no statistically significant differences between the three systems, $\left(\mathrm{X}^{2}=3.36\right),(\mathrm{p}=0.186)$, it was evidenced the presence of gaps only in groups 2 and 3 . At the middle third $\left(X^{2}=6.67\right)$ there was statistically significant difference of the seal quality of Beefill respect the others, $(p=0.036)$. At the apical third $\left(X^{2}=8.967\right)$ there was statistically significant difference of the seal quality of Beefill respect the others $(p=0.014)$. The comparison of proportions with the $\mathrm{Z}$ test, confirmed the significance of the findings. Thermoplastic technique: Beefill 2 in 1 , showed optimal quality seal in the three thirds showing statistically significant differences in middle and apical thirds $(\mathrm{p}<0.05)$. Conclusion: The thermoplastic continuous wave technique, Beefill 2 in 1 showed better sealing quality in the three thirds, since the heat-softened gutta percha and then being compacted, this runs around the entire root canal.
\end{abstract}

Keywords: Sealing quality; Root canal filling techniques; Beefill; Tagger; Lateral condensation.

\section{Artículo Original}

\author{
Marisa Jara-Castro ${ }^{1}$, Mónica Llanoz- \\ Carazas $^{1}$, Jesús Inga-Chuco ${ }^{1}$
}

\begin{abstract}
1. Segunda Especialidad Carielogia-Endodoncia, Unidad de Posgrado de la Facultad de Odontología de la Universidad Nacional Mayor de San Marcos, Perú.
\end{abstract}

\section{Correspondencia:}

Mg. Esp. Marisa Jara-Castro

Facultad de Odontología de la Universidad $\mathrm{Na}$ cional Mayor de San Marcos.

Av. Germán Amézaga s/n, Lima1, Perú.

Correo electrónico: mjarac@unmsm.edu.pe

Coautores:

Llanoz-Carazas: yizely@hotmail.com

Inga-Chuco: i.jesusivan@hotmail.com

Fecha de recepción: 10-07-14

Fecha de aceptación: 31-10-14 


\section{Introducción}

La obturación del conducto radicular y la obtención de un "sellado hermético" es citado con frecuencia como un objetivo fundamental del tratamiento de conductos $^{1,7}$.

La inhabilidad para rellenar el conducto en tres dimensiones consistirá en la formación de espacios tanto apical como coronalmente o internamente dentro de la masa de gutapercha, produciendo vías de filtración, que favorecerán el crecimiento bacteriano o la reinfección ${ }^{3,4}$.

Habitualmente la obturación de los conductos radiculares son con gutapercha que puede estar en fase alfa y beta y pueden ser convertidas una a la otra y viceversa dependiendo de la temperatura a la cual es sometida, estas transformaciones de fase están asociadas con cambios volumétricos y a fenómenos de contracción ${ }^{1,3}$ y un sellador (Endofill, fabricado en base a óxido de zinc y eugenol o cemento de Grossman) es el método biológicamente más adecuado y más seguro a largo plazo ${ }^{6}$.

Tradicionalmente se realiza la condensación lateral como la técnica estándar de obturación una vez finalizada la preparación biomecánica del conducto radicular, se selecciona el cono de gutapercha principal, se confirma su posición en la longitud de trabajo mediante la radiografía ${ }^{5,6,9}$. Se elimina el barrillo dentinario utilizando solución de EDTA al $17 \%$ por 3 minutos, se irriga y seca el conducto radicular. Se introduce el cono principal con el cemento obturador. Se selecciona el espaciador que adapte a 0.5 o $1 \mathrm{~mm}$ de la longitud de trabajo ${ }^{5,6,7}$. El espacio creado con el retiro del espaciador debe rellenarse inmediatamente con un cono accesorio de diámetro análogo al del espaciador. Este procedimiento se repite hasta que el espaciador no encuentre espacio para penetrar más allá del tercio cervical ${ }^{5,6}$.

Se han hecho repetidos intentos de mejorar la calidad de la obturación del conducto radicular con cementos y con modificaciones en la forma de suministrar la gutapercha en el conducto. Es así que Mc Spadden introdujo la técnica de termocompactación de la gutapercha, por medio de un instrumento semejante a una lima Hedström invertida, montada en contrángulo, girando en sentido horario el compactador plastifica la gutapercha reblandecida ${ }^{4,5}$. La que fue modificada por Tagger que unió la técnica de condensación lateral al uso de los compactadores Mac Spadden, desarrollando así la llamada técnica hibrida de obturación de los canales radiculares, que consiste en la realización de la condensación lateral apenas en el tercio apical ${ }^{4,5}$. Se ajusta un cono maestro en el conducto y se coloca en él con el sellador. Es necesaria la buena adaptación a la longitud del conducto y la conformación. Se realiza la condensación lateral en el tercio apical con dos o tres conos accesorios, se coloca el compactador en el conducto y se mueve apicalmente con una presión suave hasta un punto de $3-4 \mathrm{~mm}$ escasos de la longitud de trabajo o hasta que encuentre resistencia, por 10 segundos. El compactador se retira mientras aún está girando y compactando la gutapercha apical y lateralmente. Se debe considerar que el aparato de baja rotación tiene que girar como mínimo a 8,000 rpm mas no exceder las 10,000 $\mathrm{rpm}^{4,5}$. Con un giro en sentido horario $^{4,5}$. Siempre entrar y salir del interior del conducto radicular con el compactador girando ${ }^{4,5,6}$.

Con el objetivo de mejorar la homogeneidad y la adaptación de la gutapercha a las paredes del conducto. Se introdujeron las técnicas de obturación termoplastificadas las que fueron introducidas a finales de la década de los setentas, se ha sugerido que son más exitosas cuando se emplea un cemento sellador capaz de producir una película de un espesor menor a $12.7 \mu \mathrm{m}$ para humedecer la superficie de forma adecuada y en consecuencia facilitar un mejor sellado ${ }^{1,3}$, estableciendo una masa más uniforme que la que se produce con las técnicas que emplean gutapercha fría ${ }^{1}$.

Recientemente se ha introducido la técnica de obturación con gutapercha termoplastificada con Beefill 2 en 1, la cual es fabricada y distribuida por VDW GmbH en Bayer Munich Alemania, sistema combinado. Muchos odontólogos se han adecuado con los sistemas combinados, particularmente aquellos que se asocian con la condensación vertical caliente para proporcionar un sellado apical, y la inyección de gutapercha caliente con el fin de llenar los tercios cervical y medio del conducto radicular como el sistema Beefill ${ }^{1}$.

Con este nuevo aparato tenemos en una misma unidad los sistemas de obturación ${ }^{1}$ : Downpack es un transportador de calor, para realizar la condensación vertical en caliente del tercio apical, con atacadores de acuerdo al diámetro del conducto 40/03, 50/05, 60/06 que utiliza un rango de $180-250{ }^{\circ} \mathrm{C}$ de temperatura constante ${ }^{1}$, y Backfill que es un dispositivo de inyección de la gu- tapercha que nos permite seleccionar 2 niveles de velocidad para la extrusión de la gutapercha, utiliza una temperatura de 200 a $250^{\circ} \mathrm{C}$ de temperatura, para el reblandecimiento de la gutapercha, la cual es suministrada en forma de cartuchos de inyección cada $3 \mathrm{~mm}$ para luego realizar una condensación para contrarrestar este fenómeno de contracción. Este procedimiento se realizará las veces necesarias hasta rellenar el conducto radicular en toda su extensión ${ }^{1}$.

\section{El objetivo del presente estudio fue:}

- Evaluar in vitro la calidad del sellado de tres técnicas de obturación: Condensación lateral, Hibrida de Tagger y Beefill 2 en 1 por tercios radiculares en primeras premolares inferiores.

- Comparar in vitro la calidad del sellado de tres técnicas de obturación: Condensación lateral, $\mathrm{Hi}-$ brida de Tagger y Beefill 2 en 1 por tercios radiculares en primeras premolares inferiores.

\section{Metodología}

Para el estudio de investigación utilizamos 30 piezas, primeras premolares inferiores con ápice cerrado, recién extraídas, las cuales fueron sumergidas durante 4 horas en Hipoclorito de Sodio al 5.25\% para la remoción del tejido periodontal.

Se corroboró la existencia de un solo conducto, mediante radiografía, para luego uniformizarlas a una longitud de $14 \mathrm{~mm}$, longitud total de la raíz.

Se permeabilizaron los conductos radiculares con limas manuales tipo $\mathrm{K}$, No 8 y 10 de $21 \mathrm{~mm}$ de longitud y se estableció como longitud de trabajo 13 $\mathrm{mm}$ de forma visual,

20 muestras se prepararon con el sistema rotatorio MTwo, según las indicaciones del fabricante $(10 / 04,15 / 05$, $20 / 06,25 / 06,30 / 05,35 / 04)$ y 10 muestras se prepararon con la técnica corono apical de la universidad de Oregón: Gates Glidden \#3, \#2, \# 1 en tercio medio y cervical y se llegó a la lima maestra \#35 en apical, ya que la técnica original de Tagger recomienda una preparación biomecánica crown-down de la universidad de Oregón.

Todas las muestras fueron irrigadas con hipoclorito de sodio $(\mathrm{NaOCl}) 3 \mathrm{ml}$ al $5.25 \%$ y con aspiración constante, a través de todo el conducto radicular y entre cada instrumento, se eliminó el barrillo dentinario con $3 \mathrm{ml}$. de EDTA líquido al $17 \%$ durante $3 \mathrm{~min}$, neutra- 
lizado con $\mathrm{NaOCl}$, se secó el conducto con puntas de papel No 35. Las muestras se dividieron aleatoriamente en tres grupos experimentales para su posterior obturación.

El grupo 1 (10 muestras), fueron obturadas con la técnica de Condensación lateral, utilizando los conos principales del sistema MTwo 35/04 y varios conos accesorios, se seleccionó un espaciador digital no20 y utilizado con movimientos firme y oscilatorio en dirección apical tratando de presionar el cono de gutapercha principal lateralmente, el espaciador fue retirado y un cono accesorio fue introducido inmediatamente en el espacio dejado por el instrumento. Esta maniobra fue repetida hasta que el espaciador no penetrase más en el conducto hasta el límite cervical.

El grupo 2 (10 muestras), fue obturado con la técnica hibrida de Tagger, cono de gutapercha principal \#35 de conici- dad 0.02 con la ayuda de un espaciador n²0 se colocó tres conos de gutapercha accesorios, luego se seleccionó el Gutacondensor calibre 50, este fue introducido a $4 \mathrm{~mm}$ de la longitud de trabajo, prestando atención del giro en sentido horario por 10 segundos La gutapercha reblandecida fue condensada verticalmente con los condensadores Matchou color plomo (3/4 de $0.8 \mathrm{~mm})$.

El grupo 3 (10 muestras), fue obturado con la técnica termoplastificada Beefill 2 en 1, se obturó con el cono de gutapercha principal que corresponde al sistema MTwo 35/04 con un plugger para el Downpack (sellado apical) no 50/05, ayudado con un condensador Matchou color rojo $(1 / 2$ de $0.5 \mathrm{~mm})$ a nivel del tercio apical y el Backfill, sistema de inyección en el tercio medio y cervical.

El cemento obturador empleado fue Endofill, para todos los grupos. Se congelaron las muestras durante 6 horas para evitar alteraciones de la gutapercha, inmediatamente después se realizaron cortes transversales en los tercios cervical, medio y apical, con una medida de $4 \mathrm{~mm}$ por tercio, con discos de corte diamantados. Se fijaron en placas porta objetos, y fueron evaluados con el microscopio estereoscopio marca Genius 5X (Alemania).

Se tomaron las fotografías de las muestras vistas al microscopio estereoscópico, las cuales fueron analizadas por dos evaluadores calibrados que atribuyeron la cantidad de vacíos, Se realizó la calificación de la calidad de obturación mediante los criterios:

0 = sellado optimo, bloque de gutapercha homogénea con mínima cantidad de cemento sellador sin presencia de vacíos.

1 = sellado regular, bloque de gutapercha heterogénea con visible cemento obturador, y presencia de vacíos.

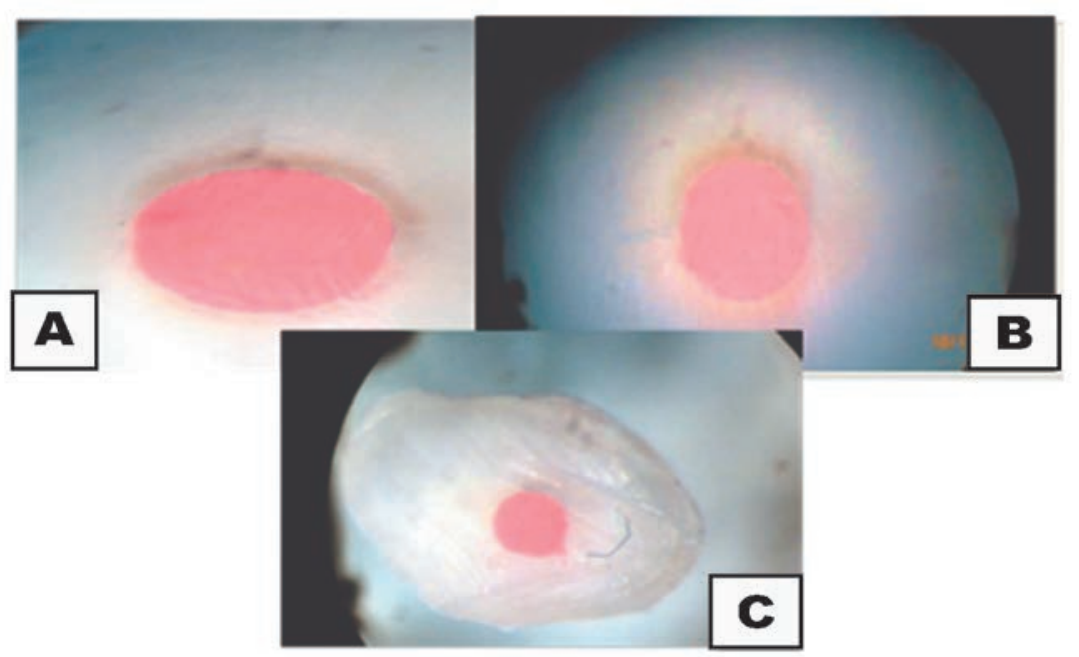

Fig. 1 Cortes transversales vistos al microscopio estereoscópico. Obturación con la técnica termoplástica Beefil 2 en 1

A). Tercio Cervical, B). Tercio Medio C). Tercio apical

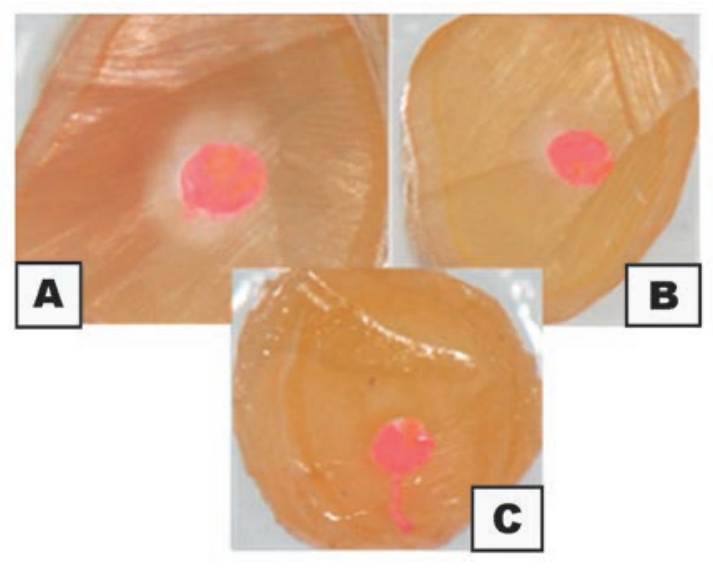

Fig 2. Cortes transversales vistos al microscopio estereoscópico. Obturación con la técnica hibrida de Tagger

A). Tercio Cervical, B). Tercio Medio C). Tercio apical

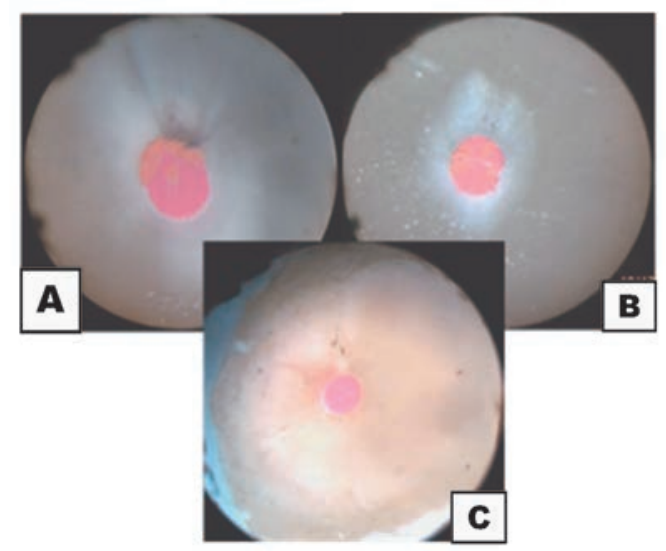

Fig.3. Cortes transversales vistos al microscopio estereoscópico. Obturación con la técnica Condensación lateral

A). Tercio Cervical, B). Tercio Medio C). Tercio apical 


\section{Resultados}

Se realizó el análisis estadístico del Chi cuadrado con un nivel de significancia de 5\%. Grupo 3 (Condensación lateral) grupo 2 (Hibrida de Tagger) grupo 1 (Beefill 2 en 1) en los tres tercios respecto a la presencia de vacíos.

A nivel del tercio cervical el resultado fue de $\mathrm{x}^{2}=3.36$ no encontrándose diferencias estadísticamente significativa para los tres sistemas $\mathrm{p}=0.186$ (Tabla 1 ).

Tabla 1. Presencia de vacíos en el tercio cervical y técnicas de obturación

\begin{tabular}{lcccccc}
\hline \multirow{2}{*}{ Tercio apical } & \multicolumn{2}{c}{$\mathrm{Si}$} & \multicolumn{2}{c}{ No } & \multicolumn{2}{c}{ Total } \\
& $\mathrm{n}$ & $\%$ & $\mathrm{n}$ & $\%$ & $\mathrm{n}$ & $\%$ \\
\hline Beefill 2en1 & 0 & 0 & 10 & 100 & 10 & 100 \\
Tagger & 3 & 30 & 7 & 70 & 10 & 100 \\
Condensación lateral & 2 & 20 & 8 & 80 & 10 & 100 \\
\hline Total & 5 & 16.7 & 25 & 83.3 & 30 & 100 \\
\hline
\end{tabular}

A nivel del tercio medio el resultado fue de $\mathrm{x}^{2}=6.67$ encontrándose diferencias estadísticamente significativa, respecto a Beefill en relación a los otros sistemas $\mathrm{p}=0.036$. Se evidenció la presencia de espacios en los grupos 2 y 3 , no así en el grupo 1 . (Tabla 2).

Tabla 2. Presencia de vacíos en el tercio medio y técnicas de obturación

\begin{tabular}{lcccccc}
\hline \multirow{2}{*}{ Tercio coronal } & \multicolumn{2}{c}{$\mathrm{Si}$} & \multicolumn{2}{c}{ No } & \multicolumn{2}{c}{ Total } \\
& $\mathrm{n}$ & $\%$ & $\mathrm{n}$ & $\%$ & $\mathrm{n}$ & $\%$ \\
\hline Beefill 2en1 & 0 & 0 & 10 & 100 & 10 & 100 \\
Tagger & 4 & 40 & 6 & 60 & 10 & 100 \\
Condensación lateral & 5 & 50 & 5 & 50 & 10 & 100 \\
\hline Total & 9 & 30 & 21 & 70 & 30 & 100 \\
\hline
\end{tabular}

A nivel del tercio apical el resultado fue $\mathrm{x}^{2}=8.9$ con una diferencia estadísticamente significativa respecto a Beefill en relación a los otros sistemas $\mathrm{p}=0.014$ (Tabla 3 ).

Tabla 3. Presencia de vacíos en el tercio apical y técnicas de obturación.

\begin{tabular}{lcrrrrr}
\hline \multirow{2}{*}{ Tercio medio } & \multicolumn{2}{c}{ Si } & \multicolumn{2}{c}{ No } & \multicolumn{2}{c}{ Total } \\
& $\mathbf{n}$ & $\%$ & \multicolumn{1}{c}{$\mathbf{n}$} & \multicolumn{1}{c}{$\%$} & \multicolumn{1}{c}{$\mathbf{n}$} & $\%$ \\
\hline Beefill 2en1 & 0 & 0 & 10 & 100 & 10 & 100 \\
Tagger & 5 & 50 & 5 & 50 & 10 & 100 \\
Condensación lateral & 6 & 60 & 4 & 40 & 10 & 100 \\
\hline Total & 11 & 36,7 & 19 & 63.3 & 30 & 100 \\
\hline
\end{tabular}

La técnica termoplástica Beefill 2 en 1, demostró un sellado óptimo, en los tres tercios, ver Fig1, Fig 2, Fig 3, observándose diferencias estadísticamente significativas en los tercios medio y apical, $\mathrm{p}<0.05$, El procesamiento y análisis estadístico de la información se realizó a través del programa estadístico SPSS versión 15.

\section{Discusión}

La técnica de condensación lateral es la más conocida debido a la simplicidad y el bajo costo. Entre las técnicas termoplastificadas la que requiere menos recursos es la hibrida de Tagger, utilizando, apenas un compactador de Mc Spadden con calibre adecuado y uno o dos conos accesorios de gutapercha. La técnica termoplástica Beefill 2 in 1 nos brinda mejores resultados de obturación pero considera mayores costos por requerir equipo e instrumental especial para dicha técnica.

Nuestro estudio mediante la observación a través del microscopio estereos- cópico demostró una mejor calidad de obturación observándose un sellado tridimensional, sin presencia de vacíos y poco cemento en la interfase con la dentina con la técnica de obturación de onda continua Beefill 2en1, estos estudios son consistentes con los estudios realizados por Lieven Robberecht ${ }^{1}$, calentando y atacando la gutapercha permite la realización de una obturación que se adapta mejor al sistema de conductos radiculares, que aumenta el ajuste apical y propulsa el cemento sellador a las variaciones anatómicas, que coincide con los resultados obtenidos por un estudio similar realizado por Yilmaz ${ }^{11}$. Considerando que la ca- lidad del sellado apical del material obturador a la pared del conducto es un factor importante para determinar el sellado tridimensional y hermético que previene la proliferación bacteriana, los resultados obtenidos en el estudio de Maniglia Ferreira ${ }^{4}$, señalan que las técnicas de obturación en frio fueron incapaces de demostrar el sellado hermético en comparación con las técnicas de obturación en caliente. La gutapercha en estado sólido no nos ofrece el sellado a las irregularidades del conducto como lo demostró Hernandez ${ }^{12}$ ya que formaban espacios dentro de la obturación del conducto coincidiendo con lo encontrado en nuestro trabajo. 
Según el estudio realizado por Hüseyin ${ }^{13}$ en la técnica en frio de condensación lateral el cemento sella el mayor porcentaje del conducto radicular, este cemento es frágil por lo tanto es más susceptible a la solubilización resultando en fallas.

Cohelo et $\mathrm{al}^{5}$ demostraron que la técnica de obturación hibrida de Tagger mostró mejores resultados referidos a un mejor sellado tridimensional de la masa obturadora en comparación con la técnica de condensación lateral con conicidad $2 \%$. Los resultados obtenidos en el presente contrastan con los resultados obtenidos por otros autores, ya que no se encontraron resultados estadísticamente significativos para la técnica de Tagger en relación con la técnica de condensación lateral.

Sería necesario realizar otros estudios a este material, en los cuales se simulen las condiciones clínicas de la boca, en cuanto a posición de los dientes en la arcada para evaluar si se logra un adecuado acceso a todas las piezas dentarias con el compactador Mc Spadden.

\section{Conclusiones}

El presente estudio demostró que:

- La técnica termoplástica Beefill 2 en 1 , presenta una mejor calidad de sellado a nivel de los tercios apical y medio en comparación a las técnicas: Condensación lateral e híbrida de Tagger.

- No existen diferencias significativas en cuanto a la calidad del sellado a nivel de los tres tercios entre las técnicas: Condensación lateral e híbrida de Tagger.

\section{Referencias bibliográficas}

1. Lieven R, et al. Qualitative evaluation of two endodontic obturation techniques: tapered singlecone method versus warm vertical condensation and injection system An in vitro study, J Oral Sci. 2012;54(1):99-104.

2. Schafer E, Erler M: Comparative study on the shaping ability and cleaning efficiency of rotary Mtwo instruments. Part I. Int Endod J. 2006;39(3):196-202.

3. Giudice-García A, TorresNavarro J. Obturación en endodoncia - Nuevos Sistemas de Obturación: revisión de literatura. Rev. Estomatol Herediana. 2011;21(3):166-174.

4. Maniglia-Ferreira C. et al Analysis of Gutta-percha's root canal Filling capacity through three different obturation techniques. Rev Sul Brasileira de Odontología. 2011;8(1):18-24.

5. Coelho et al. Estudo Comparativo entre duas técnicas obturadoras: Condensacâo Lateral vs Hibrida de Tagger. Pesq Bras Odontoped Clin Integr. Joao Pessoa 2007;7(3):217-222. Doi: 10.4034/1519.0501.0073.0004

6. Montalvan. S. et al. Comparación microscópica de la adaptación del Cono Maestro de Gu- tapercha con conicidades $2 \%$ y 6\%. Rev Estomatol Herediana. 2006;15(2):107-111.

7. Cohen, S. Vías de la pulpa, $11^{\mathrm{a}}$ ed. España: Elsevier; 2011.1004p

8. Leonardo RM. Endodoncia Tratamientos de conductos radiculares. Principios técnicos y biológicos. Sao Paulo: Editora artes médicas latinoamericanas. 2005. 1368p

9. Canalda CS. Endodoncia técnicas clínicas y bases científicas, 2 da. ed. Espańa: Elsevier. 2008. p.299301

10. Estrella C, Ciencia endodóntica, 1a ed. España: Editora Artes Médicas 2005. 623-652p.

11. Yilmaz Z. et. al. Sealing efficiency of Beefill 2in1 and System $\mathrm{B} /$ Obtura II versus single-cone and cold lateral compaction techniques. Oral Surg Oral Med Oral Pathol Oral Radiol Endod. 2009;108(6):e51-55. Doi: 10.1016/j.tripleo.2009.07.057.

12. Hernández VS. et al. Comparación de la calidad de la obturación radicular obtenida con el sistema fluido de obturación radicular v/s técnica de compactación lateral. Rev Avances en Odontoestomatología. 2008;24(4):255-260.

13. Hüseyin et al. Fracture resistance of roots filled with three different obturation techniques. Oral Surg Oral Med Oral Pathol 2012;17(3):28-32. 